\title{
Organ Donation Prediction using Trigger based Self Learning Automata
}

\author{
Sandeep Nithyanandan
}

\begin{abstract}
"Organ donation", hearing this word causes alarm bells ringing in our ears. The necessity of organ donation is a problem which is to be addressed. A lot of people suffering from many diseases requires a solid organ transplantation. But the problem is that only the demand for donors is increasing ,the supply is not met. The only resolution to this grave danger is to refine the primitive mindset of people . Even though a lot of methods have been already tested and experimented to solve the crisis of "organ donation deficiency".
\end{abstract}

A new novel computable soft solution to this psychological problem is being proposed by fixing values for a set of General Commitment Quotient. The technique uses contextual based learning algorithm to identify the mindsets and then construct a trigger based automata which has the capability to adjust the quotient values.

\section{Keywords}

Relative Contextual Learning, Global Commitment Index, Communitarian Approach, Global Knowledge Automata, Machine Learning.

\section{INTRODUCTION}

The psychological mindset of people towards organ donation is of immense value as it determines the probability of having a live donor. Maximizing the psychosocial status and wellbeing of donors both before and after organ donation thus should be an integral part in organ donation[1]. But the recent trends shows that most of the society still pose a primitive mindset regarding this. Most people think of organ donation as an out of the box activity which is of no concern to them. But the importance of the issue is addressed only when they come face to face with this threat. Everyone ought to remember that any organ donated may be the reason why another human lives. There are various factors which determine the live donor's motive behind organ donation. These include intrinsic factors and extrinsic factors. The intrinsic factors are the prime psychological reason concerning the donor. The desire to relieve the suffering of another or to act in accordance to religious conviction contribute to this . Extrinsic factors are governed by the social pressure, perceived norms, relation between donor and recipient. There is a number of psychological reason why many are reluctant to perform organ donation. The lack of selfless commitment towards the society, fear of loosing one's own body part which in turn is lack of scientific knowledge ,many false-believes regarding organ donation ,negative pressure of family members. The availability of having more live donors can be increased to a viable rate only if these psychological aspects are addressed. To address this issue is a Himalayan task. Unless these are confronted and solutions are arrived at, situation can go from bad to worse.

\author{
Jayanthan K S
}

In an era where the technology is lightening fast where most of the problems are computable, this psychological problem of organ donation has to be addressed. Using soft computing techniques to arrive at certain solutions regarding this psychosocial issue will be certainly fruitful. The technique proposed helps to identify and model the psychological approach to persuade a living donor. The algorithm is implemented to locate a living donor in a particular geographical region. This increases the chance of having a live donor for organ donation.

\subsection{Relative Contextual Learning}

Learning is a continuous process which makes humans to be different from other species. Human cerebrum implements the method of relative and contextual based learning .Here an attempt to give a different interpretation of how the human cerebrum co-ordinates it's learning activity is being done. The method proposed is based on the atomic level co-ordination of the human cerebrum's learning methodology, contextual and relative based learning. An entity is a situation specific object .The entities are mapped to form a situation centric cognitive automata whose tuples are initial state, relative link weight, cognitive transition function ,final state, feedback value. The major controlling part of the automata is the relative link weight which depends on the context of the entity and relation with other entities. The resulting weight value determines the output state.

\subsubsection{Cognitive Automata}

The automata is contextual dependent and self learning. It has the property of recursive feedback to give valuable suggestions to previous states. The automata can be further modified to time specific ,platform independent ,generalized machine for the improvement of cognitive problem solving .

Example:

The entity 'PLANT' has an ambiguous meaning based on Biological plant and Industrial power plant. The cognitive automata learns the situation and relates the two entities using relative link weight to arrive at a conclusion .It can infer different relationships between entities throughout the learning cycle .So the above proposed Relative Contextual Learning give insights to a new generation of computing where machines interpret the data and provide information without human intervention.

\section{LITERATURE SURVEY}

The shortage for organ donors is a psychological problem .Much depends on how people presume this scenario as a crisis[14]. Various suggestions have been made to respond to the high demand of organs by paying for them. There are many undesirable effects to this including commodification, injustice, cost So in order to tackle the problem various psychological approaches have been proposed earlier. An exemplary approach to this is a Communitarian Approach[2]. The communitarian approach entails changing the predefined 
moral culture and mindset of members of society regarding organ donation. The approach requires much greater and deeper efforts than sharing information and making public service announcement. The mindset of society regarding organ donation has to be analyzed theoretically and then precise efforts has to be made[4]. The core of this approach entails changing people's preference by means of moral persuasion, community recognition and appreciation of good conduct and gentle chiding of those who think it is not right. The key is to convert the existing predisposition into active preference. A survey conducted indicates around $85 \%$ support organ donation, but only $35 \%$ are willing to donate their organs[11]. This indicates a positive shift of the tide. The means adopted by this approach involves Moral Dialogues. The community people live in is highly moral oriented .Moral dialogues are social processes that involves merely not talking about facts and figures ,but intensive discussion of the values of those engaged in the dialogues. Such an approach can be of great help as it makes the listener understand the value of another person's life. To significantly change the moral culture surrounding organ donation a full-blown moral dialogue about the fact that many are neglecting to donate organs which are of no use to them. By doing so the hearer understand the need of organ donation and can persuade him to do it[16]. Thus communitarian approach serves as a model for an approach to organ donation.

The world today embraces the computer era. To every problem today there is a computable solution. Automata theory can be successfully applied to solve the general psychological problems. The cellular automata can be applied in solving such issues as it's each state is directly affected by it's neighboring states. The stimulus based response (S-R) of human being can be modeled into an automata. This model can then be successfully applied in solving many psychological problem. Finite Automata can be used to represent mental models[10]. By doing so it is able to predict the way a human being thinks and behaves in a situation. This helps to solve merely any psychological problem as this enables to deal with the different situations that one come up against. So an automata theory is a computable solution that can be successfully applied to solve the organ donation crisis[8]. These methods gives an insight to the various ways a problem can be represented in a computable manner. As a result any know problem nowadays can be solved computably.

\section{TERMINOLOGY}

The term that is going to be defined here is General Commitment Index(GCI).

GCI can be defined as the overall commitment of a person to the society he lives in. The GCI is determined by various factors which are dependent to the individual itself and to the society. The GCI gives a better view and helps to understand about how a person views the society he live in and how the society views and understands him. The various indices which governs GCI are:

- Social Awareness Index(SAI):SAI can be defined as the self commitment of a person to the society he lives in. It tells how a person sees himself attached to a society and how he values the society.SCI is also about how a person wants to be a part of the development of the society.
- Cultural Value Index(CVI) :CVI can be defined as the cultural values a person beholds. It is the belief in the customs and functions of a culture .The CVI can be determined based on the religion of a person also.

- Myth Co-efficient Index(MCI): MCI refers to the various myths that a person has regarding a particular subject, here it is organ donation. It may be based on religious beliefs and the knowledge that the person beholds.

- Peer Pressure Index(PPI):PPI is the pressure that a person is being exerted upon by his peers and family. PPI has importance value in case of organ donation because a person's willingness to donate can be largely factorised upon PPI.

- Scientific Knowledge Index(SKI):SKI is the scientific knowledge that a person has. It plays an important role in case of organ donation. The person with proper SKI know the need for organ donation and what it means to be a donor.

- Economic Background Index(EBI):EBI is also a factor because the economic stability of a person. It can also help in determining whether he can be a donor or not.

\section{PROPOSED METHOD}

In this proposal the GCI values are tabulated on the basis of a survey conducted at parappur around 500 homes .From the survey threshold values of GCI co-efficients that supports organ donation was obtained. The threshold values were interpreted to attain the stable index values. On the basis of the stable index values a time and situation based Global Knowledge Automata (GKA) is created. The stable index values of the above automata are depicted in the table 1.The tuples of the automata includes the stable index values as the states , triggered transition function ,time and situation. The triggered transition function facilitates the transition from one state to another state with the help of The GKA is used as the reference automata and is shown in the figure below.

The machine inferred automata is called Person Specific Automata(PSA). The PSA is specific to each person and may differ in it's values. It is constructed on the basis of the values which were inferred by the machine from the user. The tuples of the person specific automata are stable or unstable index values as the states, triggered transition function, time and situation. The tuples time and situation plays a major role in organ donation prediction. After the construction of the PSA the learning length $\mathrm{D}$ is calculated as the difference between the transition values of the GKA and PSA. It is mathematically notated as :

$\mathrm{D}=d\left(\delta\left(Q_{i}->Q_{i+1}\right)\right)-d\left(\delta\left(Q_{i}^{\prime}->Q_{i+1}^{\prime}\right)\right)--1$

If the learning length is less than zero then the person specific automata is compatible for organ donation .The other case entails the updation of the triggered transition function $\delta$ ' as the sum of PSA and learning length D. It is mathematically notated as

$\delta^{\prime}=d\left(\delta\left(Q_{i}^{\prime}->Q^{\prime}{ }{ }^{++1}\right)\right)+D$

Simultaneously the value of learning length $\mathrm{D}$ is stored in a hash array $\mathrm{A}$. The values of the hash array $\mathrm{A}$ is interpreted with a symbolic knowledge based table constructed with the help of PROLOG. The values were fed 
into an Automata so that they can be represented in a computable method and a solution can be arrived.

\begin{tabular}{|ll|l|}
\hline \multicolumn{2}{|l|}{ INDEX } & STABLE VALUE \\
\hline$\bullet$ & $\begin{array}{l}\text { Social Awareness } \\
\text { Index(SAI) }\end{array}$ & 0.8 \\
\hline$\bullet$ & $\begin{array}{l}\text { Cultural Value } \\
\text { Index(CVI) }\end{array}$ & 0.7 \\
\hline$\bullet$ & $\begin{array}{l}\text { Peer Pressure } \\
\text { Index(PPI) }\end{array}$ & 0.6 \\
\hline$\bullet$ & $\begin{array}{l}\text { Scientific } \\
\text { Knowledge } \\
\text { Index(SKI) }\end{array}$ & 0.5 \\
\hline$\bullet$ & $\begin{array}{l}\text { Economic } \\
\text { Background } \\
\text { Index(EBI) }\end{array}$ & 0.4 \\
\hline$\bullet$ & $\begin{array}{l}\text { Myth Co-efficient } \\
\text { Index(MCI) }\end{array}$ & 0.3 \\
\hline
\end{tabular}

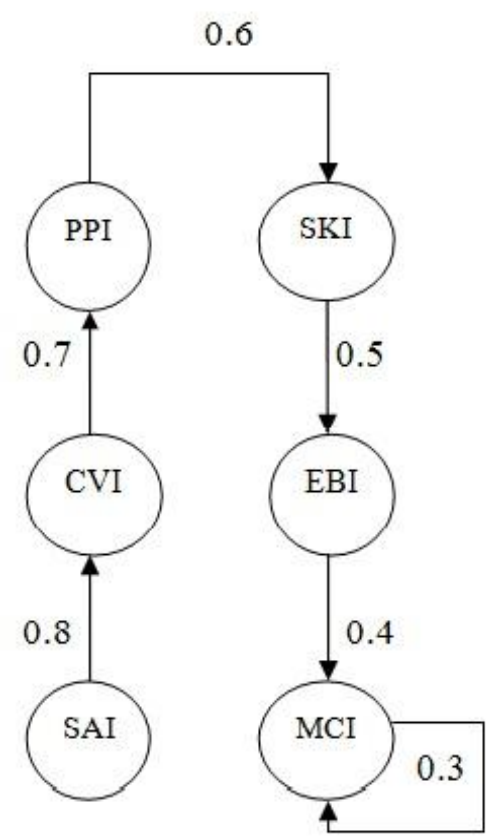

Fig 1 : Global Knowledge Automata

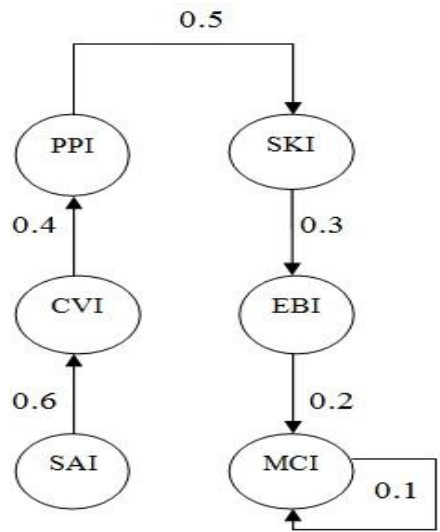

Fig 2. Person Specific Automata

\section{PROPOSED ALGORITHM:}

\section{INPUT:}

The set of GCI and PSI values.

\section{OUTPUT:}

Suggestions for improving the PSI to attain stable GCI values.

- Create a Global Knowledge Automata on basis of GCI values.

GKA can be mathematically defined on the tuple values of $\left\langle P, P_{0}, P_{f}, \delta, T, S>\right.$.

Where $P$ is the set of GCI index values \{SAI, CVI ,PPI,SKI, EBI,MCI\}.

$\delta$ is the triggered transition function.

$T$ is the time specific state.

$S$ is the situation specific state.

- Create a Person Specific Automata inferred by the machine from the user.

Where $P^{\prime}$ is the set of person specific index(PSI) values \{SAI, CVI,PPI,SKI, EBI,MCI\}.

$\delta^{\prime}$ is the triggered transition function.

$T$ is the time specific state.

$S$ is the situation specific state.

- $\quad$ The learning length $D=d\left(\delta\left(Q_{i} \rightarrow Q_{i+1}\right)\right)-d(\delta$ $\left.\left(Q_{i}^{\prime} \rightarrow Q_{i+1}^{\prime}\right)\right)$

If $D<0$, then flag $=1$, else flag $=0$.

If flag $==0$, then update the learning length $\delta^{\prime}=d\left(\delta\left(Q_{i}\right.\right.$ $\left.\left.->Q^{{ }^{\prime}}{ }^{+1}\right)\right)+D$.

Simultaneously store the value of $D$ in a hash array $A$.

- $\quad$ The value of hash array $A$ is interpreted using a symbolic table and suggestions to improve the PSI values. 


\section{PROPOSED SYSTEMARCHITECTURE}

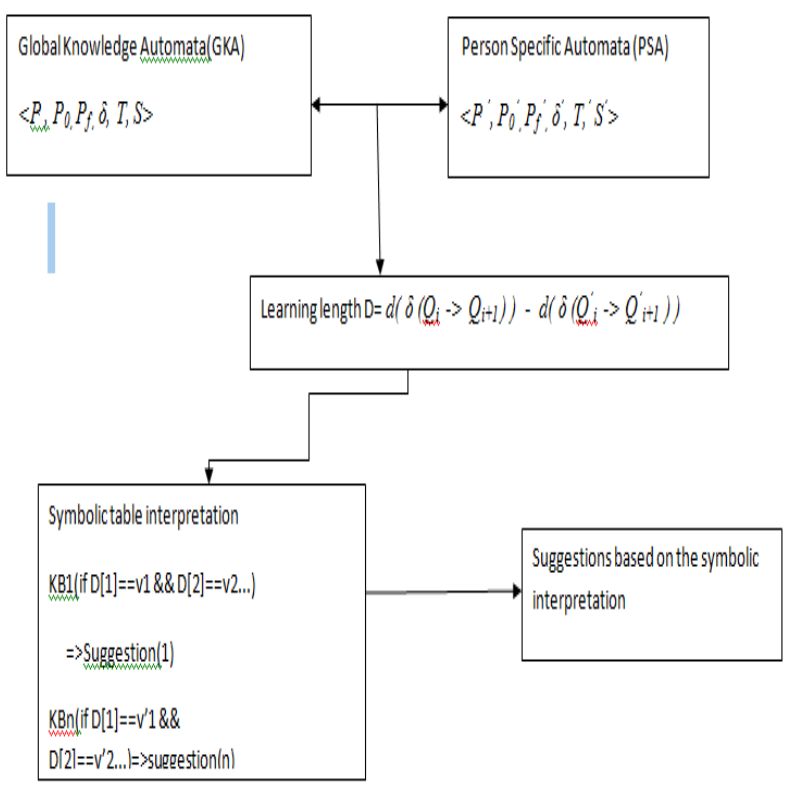

Fig 3: Schematic representation of proposed system architecture

\section{IMPLEMENTATION}

The system is implemented with the help of JAVA ,INTERPROLOG AND SWI PROLOG. The automata is trained with the 500 data sets collected from the survey conducted .The Global Knowledge Automata is constructed with stable value indices which is then visualized using Java interface .The Person Specific Automata is constructed by the machine inferring from the user data. The symbolic knowledge base for the learning distance $\mathrm{D}$ is created using SWI PROLOG. The system gives $92.8 \%$ accuracy on the suggestions for organ donation.

\section{RESULT AND COMPARISONS}

About $92.8 \%$ accuracy was obtained and two tables has been tabulated. The two tables indicated below is tabulated by

1:) Comparing the self learning automata technique over traditional machine learning technique. The results obtained showed that the Self Learning Automata showed greater accuracy than the traditional machine learning technique.

Table 1 :Comparion of Self learning Automata Technique over Traditional Machine Learning Technique

\begin{tabular}{|l|l|l|}
\hline $\begin{array}{l}\text { NUMBER OF } \\
\text { DATA SET }\end{array}$ & $\begin{array}{l}\text { SELF- } \\
\text { LEARNING } \\
\text { AUTOMATA } \\
\text { ACCURACY(\%) }\end{array}$ & $\begin{array}{l}\text { TRADITIONAL } \\
\text { MACHINE } \\
\text { LEARNING } \\
\text { TECHNIQUE } \\
\text { ACCURACY(\%) }\end{array}$ \\
\hline 100 & 81.5 & 79 \\
\hline 200 & 84.2 & 81 \\
\hline 300 & 87.5 & 85.7 \\
\hline 400 & 90 & 86 \\
\hline 500 & 92.8 & 87.5 \\
\hline
\end{tabular}

2:) The below table shows the accuracy of prediction when the PSA values were given as input.

Table 2 : Organ Donation Prediction Accuracy

\begin{tabular}{|c|l|l|l|}
\hline GKA & PSA & LEARNING & PREDICTION \\
VALUES & VALUES & LENGTH & ACCURACY(\%) \\
\hline SAI -0.8 & 0.5 & 0.3 & 82.5 \\
& 0.7 & 0.1 & 91.2 \\
\hline CVI -0.7 & 0.5 & 0.2 & 86.7 \\
& 0.4 & 0.3 & 82.5 \\
\hline PPI -0.6 & 0.3 & 0.3 & 82.5 \\
& 0.2 & 0.4 & 76.7 \\
\hline SKI -0.5 & 0.2 & 0.3 & 82.5 \\
& 0.4 & 0.1 & 91.2 \\
\hline EBI -0.4 & 0.1 & 0.3 & 82.5 \\
& 0.3 & 0.1 & 91.2 \\
\hline MCI - 0.3 & 0.1 & 0.2 & 86.7 \\
& 0.2 & 0.1 & 91.2 \\
& & & \\
\hline
\end{tabular}

\section{CONCLUSION}

The proposed method give an insight for solutions to psychological problems using computational techniques. The proposed Global Knowledge Automata and Person Specific Automata give new explorations to computable psychological modeling. The Relative Contextual Learning proposed in this paper give new insights to machine learning techniques. The above implemented algorithm is a state -of- art approach to solve organ donation crisis. The GKA can be further implemented on solving psychological related problems.

\section{REFERENCES}

[1]: Alexandra Melnikova , Boris Melnikov . A "New Algorithm of Constructing the Basis Finite Automaton".

[2]:Amitai Etzioni. "Organ Donation : A Communitarian Approach".

[3]: Andrea F. DiMartini ,Galen E. Switzer , Larissa Myaskovsky , Mary Amanda Dew ,Megan CrowelyMatoka . "Psychosocial Aspects of Living Organ Donation".

[4]: Andrei Holman . "Psychosocial Determinants of Organ Donation Intentions and their Relevance for Public Campaigns" . Postmodern Openings, 2013, Volume 4, Issue 1, March, pp:9-27 .

[5]: Angelo Montanari, Davide Bresolin ,Gabriele Puppis . "A Theory of Ultimately Periodic Languages and Automata with an Application to Time Granularity".

[6] : A.S. Daar , P. Marshall . "Cultural and psychology in organ transplantation".

[7]: Axel Buchner, Joachim Funke . "Finite-state Automata: Dynamic Task Environments in Problem - solving Research". The Quarterly journal of Experimental Psychology , 1993,46A(1) 83-118.

[8]: Christian W. Omlin , C. Lee Giles . "Constructing Deterministic Finite-State Automata in Recurrent Neural Networks". Journal of the ACM, Vol. 43, No. 6, November 1996, pp. 937 \pm 972 .

[9]: Diana Allardyce, Magi Sque, Sheila Payne, Tracy Long "Why relatives do not donate organs for transplants: 
'sacrifice' or 'gift of life ? ". Journal of Advanced Nursing 61(2), 134-144doi: 10.1111/j.13652648.2007.04491.x.

[10]:D. Scott , M.O. Rabin. " Finite Automata and their Decision Problems”. IBM Journal . April 1959.

[11]: Joyce A. Griffin. “ Organ Donation - New Strategies for finding organs".

[12]: Margus Veanes , Wolfgang Grieskamp , Wolfram Schulte , Yuri Gurevich . "Generating Finite State Machines from Abstract State Machines".

[13]: Markus Roggenbach. "Determinization of BuchiAutomata".

[14]: Michael Marcangelo, Thomas W. Heinrich. "Psychiatric Issues in Solid Organ Transplantation".

[15]:Sarah Bunn. "Organ Donation and Transplants".

[16]: Susan E. Morgan , Lisa V. Chewning, Tyler R. Harrison . "The Challenges of Social Marketing of Organ
Donation: News and Entertainment Coverage of Donation and Transplantation" .Health Marketing Quarterly, Vol. 25(1/2).\# 2008 by The Haworth Press.

[17]:Yoav Freund, Michael Kearns, Dana Ron, Ronitt Rubinfeld, Robert E.Schapire, Linda Sellic. "Efficient Learning of Typical Finite Automata from Random Walks".

[18]: Cem Ünsal. "Stochastic Learning Automata".

[19]:"Organ Donation And Transplantation Policy Options at EU Level".

[20]:“Organ And Tissue Donation By Living Donors”. European Commission

[21]:“Organ donation and transplantation". Special Eurobarometer 333a.

[22]: "Increasing Organ Donation in NSW". Discussion paper. 